\title{
MEAN MAGNITUDES AND COLOURS OF SIX CEPHEIDS \\ IN THREE RED GLOBULAR CLUSTERS OF THE LARGE MAGELLANIC CLOUD
}

\author{
SERGE DEMERS* \\ Laurentian University, Sudbury, Ont., Canada
}

\begin{abstract}
Photographic $B$ and $V$ light curves are determined for six variables, with periods longer than one day, in and near NGC 1751, NGC 1953, and NGC 2121. New photoelectric sequences are used to calibrate the plates. The mean magnitudes and colours of these variables are similar to the magnitudes and colours of Classical Cepheids of the same period. The photometric properties of these variables are unlike Population II Cepheids in the Galaxy but are comparable to field Cepheids of the Large Magellanic Cloud.
\end{abstract}

\section{DISCUSSION}

Feast: Can one estimate the possibility of these Cepheids being Magellanic Cloud field stars (not cluster members)?

Demers: This has been done by Hodge and Wright. They compared the number of variables near their red clusters and in some fields of the LMC. They estimated that more variables were near the red clusters per unit area than in the outer fields. That is the major reason why they believe in the cluster membership of these variables.

* Visiting Astronomer, Cerro Tololo Inter-American Observatory.

** Complete details of this work will be published elsewhere. 